\title{
A Two-Microphone Method for the Determination of the Mode Amplitude Distribution in High-Frequency Ducted Broadband Sound Fields
}

\author{
P. F Joseph \\ Institute of Sound and Vibration Research. University of Southampton, Southampton SO17 1BJ, UK.
}

This paper describes a measurement technique that allows the modal amplitude distribution to be determined in ducts with mean flow and reflections. The method is based only on measurements of the acoustic pressure two-point coherence at the duct wall. The technique is primarily applicable to broadband sound fields in the high frequency limit and whose mode amplitudes are mutually incoherent. The central assumption underlying the technique is that the relative mode amplitude distribution is independent of frequency. The twomicrophone method proposed in this paper is also used to determine the transmitted sound power and far field pressure directivity.

PACS numbers:

Keywords: $\quad$ Broadband noise, duct acoustics, measurement techniques

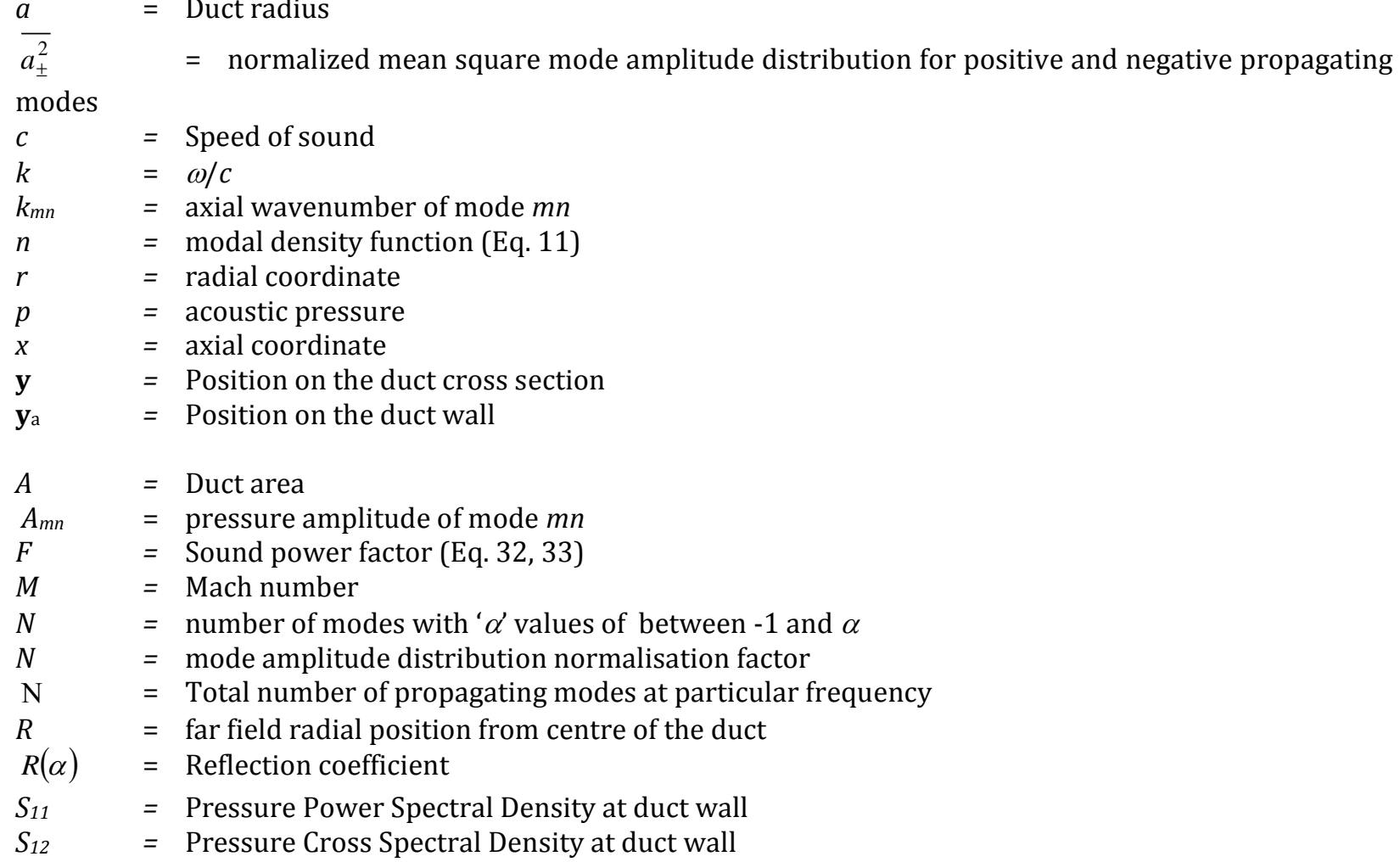

$=$ normalized mean square mode amplitude distribution for positive and negative propagating

$=$ Speed of sound

$=\omega / c$

axial wavenumber of mode $m n$

modal density function (Eq. 11)

acoustic pressure

$=$ axial coordinate

$=$ Position on the duct cross section

Position on the duct wall

Duct area

pressure amplitude of mode $m$

= Mach number

$=$ number of modes with ' $\alpha$ ' values of between -1 and $\alpha$

$=$ mode amplitude distribution normalisation factor

= Total number of propagating modes at particular frequency

= far field radial position from centre of the duct

$=$ Pressure Power Spectral Density at duct wall

$=$ Pressure Cross Spectral Density at duct wall

\section{Nomenclature}

Duct radius 


$\begin{array}{llll}45 & S_{f f}(\omega, \theta) & =\text { Pressure PSD of far field radiation } \\ 46 & S(\omega) & = & \text { source term }(E q .7) \\ 47 & W(\omega) & = & \text { total transmitted sound power spectrum } \\ 48 & W^{ \pm}(\omega) & = & \text { Upstream and downstream transmitted power } \\ 49 & \alpha & = & \text { cuton ratio (Eq. 3) } \\ 50 & \beta & = & \text { Lorentz factor } \\ 51 & \delta & = & \text { reflection factor } \\ 52 & \kappa_{\mathrm{mn}} & = & \text { transverse modal wavenumber } \\ 53 & \theta & = & \text { circumferential coordinate } \\ 54 & \rho & = & \text { mass density of air } \\ 55 & \omega & = & \text { angular frequency } \\ 56 & \hat{\omega} & = & \text { non-dimensional frequency (Eq. 16) } \\ 57 & \omega_{m n} & =\text { natural frequency of mode mn } \\ 58 & \theta & = & \text { polar coordinate of far field observer } \\ 59 & \Gamma_{m n} & =\text { Mode normalization factors } \\ 60 & \Omega^{ \pm} & =\text {Sound power factors (Eq. }(29,30) \text { ) } \\ 61 & \Psi_{m n} & =\text { ortho-normal mode shape function } \\ 62 & \psi_{12} & =\text { complex coherence function (Eq. 22) }\end{array}$

This paper deals with a measurement technique that allows the distribution of mode amplitudes to be determined as a function of their cuton ratio in a hard walled duct containing an axial uniform mean flow. Measurement of this mode distribution function then allows for estimation of the sound power transmitted along the duct and of the directivity of the far field pressure radiated from the end of the duct. The unique feature of this method is that it requires only measurements of the coherence function between two closely spaced acoustic pressure measurements made at the duct wall and is applicable in the high frequency limit where conventional mode detection methods fail since they require an unrealistically large numbers of microphones. A preliminary version of this method was presented by the author in Joseph ${ }^{1}$. radiates from the open end, causing annoyance to observers living in close proximity. Particular examples include the noise propagating along a modern turbofan aeroengine, the sound field propagating up large chimney stacks and the noise propagating along ventilation duct systems. A particular characteristic of these 
broadband sound fields is that, at sufficiently high frequency, they comprise numerous propagating duct modes, each of which has different amplitudes and phase speed. Knowledge of the pressure modal amplitudes is important in the design of an effective sound absorbing liner for determining the sound power transmitted along the duct and the far field radiation directivity pattern. However, as frequency increases the number of modes that can propagate along the duct increases roughly as the square of the frequency. In large ducts therefore, even at quite moderate frequencies, the number of duct modes that are able to propagate can readily exceed several hundred.

One of the first attempts at determining mode amplitudes from measured acoustic pressure data in a duct was by Moore ${ }^{2}$ who describes a method for determining the circumferential and radial modes at the inlet of a duct. A similar principle is described by Thomas et $\mathrm{al}^{3}$, which then extended by extended by Farassat, Nark, and Russel ${ }^{4}$. Their approach consists in applying Fourier analysis of the far-field pressure in the azimuthal direction to deduce the spinning mode amplitudes. The radial modes within each spinning mode were deduced by inverting a system of linear equations relating the pressure measurements to the mode amplitudes. The conditioning of the matrix to be inverted was assessed and some regularisation techniques applied to reduce the errors introduced by the poorly conditioned matrix. However, their technique requires roughly twice the total number of propagating modes to be inverted and is restricted to tonal noise. Since these early papers there have been numerous variants on this approach for deducing the mode amplitudes at a single frequency, such as that proposed by Lewy 5 for deducing the far field radiation.

\section{Relatively few measurement techniques for the detection of mode amplitudes of the broadband sound} field can be found in the literature, in which the acoustic signal is distributed over a frequency bandwidth and whose mode amplitudes are generally uncorrelated. Two such techniques have been presented by Enghardt et $\mathrm{al}^{5}$ and Tapken ${ }^{6}$ that requires cross-spectral measurements to be made between a number of microphones and a single reference microphone at the duct wall. However, a similar number of microphones are needed as the methods proposed for single frequency radiation, which is prohibitively large at moderate to high frequencies $(k a>20$, where $k$ is the free space wavenumber and $a$ is the duct radius). 
Another approach is that adopted by Abom ${ }^{7}$ who uses a system of transfer function measurements made between a number of microphone pairs in the duct. Again, as many measurements as modes are needed to solve the full system of equations and determine all mode amplitudes.

Lowis et $\mathrm{al}^{8}$ have proposed a pragmatic approach to determining the mode amplitude distribution. It is based on the hypothesis that different modes with the same propagation angle have similar sound transmission and radiation characteristics. It is therefore sufficient to determine the modal amplitudes as a function of propagation angle modes rather than the usual mode indices $(m, n)$. The approach by Lowis et al uses an axial array of equally spaced microphones along the duct wall to perform beamforming of the acoustic pressure at the duct to to estimate the in-duct noise directivity. This in-duct directivity may then be used to infer the far field directivity.

Rather than providing information about the mode amplitude for the different mode orders $(m, n)$, the technique provides information about the distribution of mode amplitudes versus in-duct propagation angle. For the intended applications listed above, this limitation presents no difficulty as it has long been recognized that modes with the same propagation angles possess near identical transmission and radiation characteristics 9 .

\section{MODAL TRANSMISSION}

Consider a hard-walled cylindrical duct of finite-length, as sketched in Fig 1 below, containing an axial uniform mean flow moving in the positive $x$ direction with flow speed $c M(M>0)$, where $c$ is the sound speed and $M$ is the mean flow Mach number. A point on the duct cross section is represented by $\mathbf{y}=(r, \theta)$ and $x$ denotes the axial distance along the duct relative to some arbitrary origin. Two microphones mounted flush to the duct wall, separated axially by a distance $\Delta x$, are used to detect the acoustic pressure. The objective here is to deduce the distribution of mode amplitudes in the duct using the acoustic pressure information at the two microphones. 
136

137

142

143

144

145

146

147 where $\frac{\bar{D}}{D t}=\frac{\partial}{\partial t}+c M \frac{\partial}{\partial x}$ is the convected derivative operator associated with the mean flow velocity 148

150

151

152

153

154

155

Figure 1. Semi-infinite, hard walled unflanged circular duct with associated co-ordinate system. Two microphones mounted flush to the duct wall, separated axially by a distance $\Delta x$, are used to detect the acoustic pressure.

The sound field $p(x, \mathbf{y})$ in the duct satisfies the convected homogeneous wave equation,

$(c M, 0,0)$ in the $(x, y)$ coordinate system and $c$ is the sound speed in the quiescent medium. Above its cutoff frequency, at a single frequency $\omega$, a single mode of pressure amplitude $A_{m n}$ is described by

where the superscript '+; refers to modes propagating in the direction of flow and '-' to modes propagating in the opposite direction to the flow. Equation (2) in Eq. (1) gives

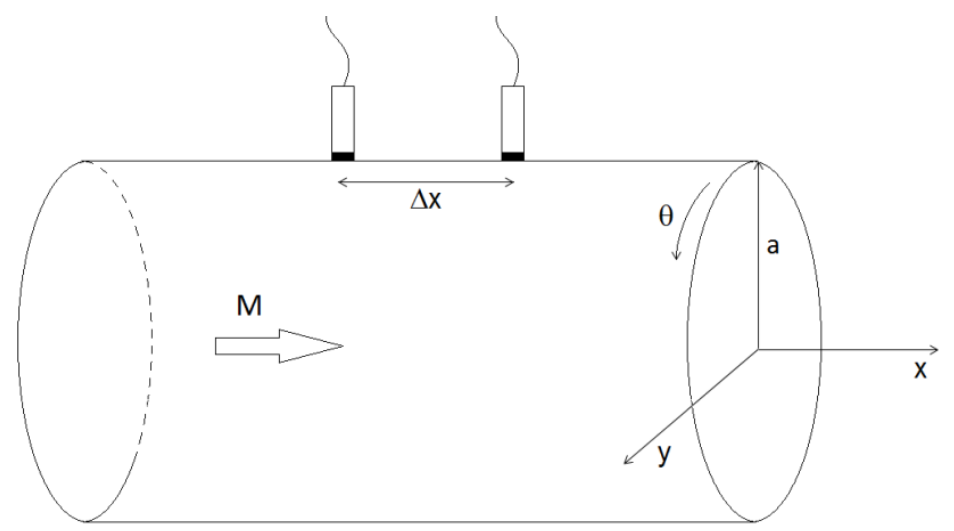




$$
k_{m n}^{ \pm}=\left(\frac{\alpha_{m n}^{ \pm}-M}{\beta^{2}}\right) \frac{\omega}{c}, \quad \alpha_{m n}^{ \pm}= \pm \sqrt{1-\left(\kappa_{m n} / k\right)^{2} \beta^{2}}
$$

where $\beta^{2}=\left(1-M^{2}\right), k=\omega / c$ where $\omega$ is the angular frequency and $c$ the speed of sound, and $\kappa_{m n}$ are a set of eigenvalues that are characteristic of the duct cross section such that the corresponding mode shape functions $\Psi_{m n}$, defined by $\left(\nabla_{\perp}^{2}+\kappa_{m n}^{2}\right) \Psi_{m n}(\mathbf{y})=0$, also satisfy the duct-wall boundary conditions and the normalization condition $A^{-1} \int_{A}\left|\Psi_{m n}(\mathbf{y})\right|^{2} d A(\mathbf{y})=1$. The parameter $\alpha_{m n}^{ \pm}$, which we shall call the cut-on ratio, is central in what follows, and takes values between $\alpha_{m n}^{ \pm}=0$ precisely at the modal cutoff frequency $\omega=\omega_{m n}=\kappa_{m n} c\left(1-M^{2}\right)^{-\frac{1}{2}}$, and tends to $\left|\alpha_{m n}^{ \pm}\right|=1$ as $\omega / \omega_{m n} \rightarrow \infty$, corresponding to modes well above cuton.

164 direction (against the flow) are represented by $\alpha_{m n} \leq 0$.

168

The in-duct sound field at any position in the duct cross section $\mathbf{y}=(r, \theta)$, axial position $x$, and frequency $\omega$, can be expressed as the sum of modal components propagating in the direction of flow $p_{m n}^{+}$, and modes propagating opposite to the direction of flow $p_{m n}^{-}$,

172

$$
p(\mathbf{y}, x)=\sum_{m=-\infty}^{\infty} \sum_{n=0}^{\infty}\left[p_{m n}^{+}(\mathbf{y}, x)+p_{m n}^{-}(\mathbf{y}, x)\right]
$$




\section{ACOUSTIC PRESSURE AT THE DUCT WALL}

178

180

181

182

183

184

185

$$
E\left\{\left|A_{m n}^{ \pm}(\omega)\right|^{2}\right\}=S(\omega) \overline{a_{ \pm}^{2}}\left(\alpha_{m n}\right)
$$

199

The acoustic pressure cross spectrum between two points separated axially along the duct wall $\mathbf{y}_{a}=(r=a, \theta)$, at axial distances $x_{1}$ and $x_{1}+\Delta x$, may written as,

$$
S_{12}\left(\omega, \mathbf{y}_{a}, x_{1}, x_{2}\right)=\frac{\pi}{T} E\left\{p\left(\mathbf{y}_{a}, x_{1}\right) p^{*}\left(\mathbf{y}_{a}, x_{1}+\Delta x\right)\right\}
$$

where $E\{\}$ denotes the expectation and the acoustic pressures refer to Fourier Transforms of the pressure time series taken over a time duration $T$. For incoherent excitation of the sound field we treat the mode amplitudes as uncorrelated random variables so that $E\left\{A_{m n} A_{m^{\prime} n^{\prime}}^{*}\right\}=0$. We further assume that the incident and reflected pressure will be incoherent since each mode will reflect with a different phase shift and hence, $E\left\{\sum_{m, n}\left[A_{m n}^{+}(\omega) A_{m n}^{-*}(\omega)\right]\right\}=0$. Substituting Eqs (2) and (4) into Eqs (5) and invoking the uncorrelated incident and reflected pressure assumption above leads to,

Work by Rice ${ }^{9}$, and more recent work by Joseph et al ${ }^{10}$, have shown that there are a physically important class of source distributions for which the relative mode amplitude distribution is independent of frequency and only a function of the cut off ratio $\alpha_{m n}$ (equivalently, mode propagation angle", see equation (25) below). Well known examples include a uniform distribution of monopole sources, axial dipole sources and equal energy per mode ${ }^{10}$. In these, and many other source distributions, we may write,

$$
S_{12}(\omega, \mathbf{y})=\sum_{m, n}\left[E\left\{\left|A_{m n}^{+}(\omega)\right|^{2}\right\} \mathrm{e}^{i k_{m n}^{+} \Delta x}+E\left\{\left|A_{m n}^{-}(\omega)\right|^{2}\right\} \mathrm{e}^{i k_{m n}^{-} \Delta x}\right] \Psi_{m n}^{2}(\mathbf{y})
$$


where $S(\omega)$ is the frequency-dependent source strength with dimensions of pressure squared per unit

201 frequency and $\overline{a_{ \pm}^{2}}\left(\alpha_{m n}\right)$ specifies the relative distribution of non-dimensional mean square mode amplitudes,

202 which depends only on $\alpha_{m n}$. A list of some physically important examples can be found in Joseph et al ${ }^{10}$. The

203 assumption of the separability of $E\left\{\left|A_{m n}^{ \pm}(\omega)\right|^{2}\right\}$ into a purely frequency-dependent term $S(\omega)$ and a mode

204 distribution term $\overline{a_{ \pm}^{2}}\left(\alpha_{m n}\right)$ (which controls the spatial variation of the sound field) is central to the validity of

205 the technique. The split between the two terms in Eq. (7) is essentially arbitrary. For reasons that will become

206 clear below, we define $\overline{a_{ \pm}^{2}}\left(\alpha_{m n}\right)$ to have the normalization property,

207

208

$$
\sum_{m, n}\left[\overline{a_{+}^{2}}\left(\alpha_{m n}\right)+\overline{a_{-}^{2}}\left(\alpha_{m n}\right)\right]=1
$$

We now denote the amplitude of waves propagating against the direction of flow (i.e., reflected modes in this case) by negative argument $\alpha$ so that

$$
\overline{a_{+}^{2}}\left(\alpha_{m n}\right)=\overline{a^{2}}\left(\alpha_{m n}\right), \quad \quad \alpha_{m n} \geq 0
$$

$$
\overline{a_{-}^{2}}\left(-\alpha_{m n}\right)=\overline{a^{2}}\left(\alpha_{m n}\right), \quad \alpha_{m n} \leq 0
$$

In the high frequency limit $(k a=\omega a / c>10$ has been found to be sufficient from our simulations, where $a$ is the duct radius), we may treat $\overline{a^{2}}(\alpha)$ as a continuous variable so that the discrete summation over $\overline{a^{2}}\left(\alpha_{m n}\right)$

217 in Eq. (6) may be replaced by an integration over $\alpha$. The normalization condition of Eq. (8) may therefore be 218 written as

$$
\int_{-1}^{1} \overline{a^{2}}(\alpha) n(\alpha) d \alpha=1
$$


222 where $n(\alpha)$ the modal density function introduced to take into account the distribution of modes across their 223 range of $\alpha$-values, defined by,

$$
n(\alpha)=\left.\frac{N(\alpha+\delta \alpha)-N(\alpha)}{\mathrm{N} \delta \alpha}\right|_{\lim \delta \alpha \rightarrow 0}, \quad \int_{-1}^{1} n(\alpha) d \alpha=1
$$

where $N(\alpha)$ is the number of modes with ' $\alpha$ values of between -1 and $\alpha$ and $\mathrm{N}$ is the total number of

propagating modes at frequency $k a$, i.e., $\mathrm{N}=N(1)=\int_{-1}^{1} N(\alpha) d \alpha$. Rice has shown that in a cylindrical duct with uniform mean flow, the total number of propagating modes $\mathrm{N}$ takes the high-frequency limiting value ${ }^{9}$,

$$
\mathrm{N} \rightarrow\left(\frac{1}{2} k a / \beta\right)^{2}, \quad k a / \beta \rightarrow \infty
$$
function $n$, is given by,

$$
n(\alpha)=|\alpha|
$$

Note that Eq. (13) differs by a factor of $1 \frac{1}{2}$ from the expression originally presented by Joseph et al ${ }^{10}$, which assumes a distribution a modes propagating from one direction only. Equation (13) indicates a scarcity of modes that are just cut-on $(\alpha \approx 0)$ compared with a higher population of modes that are well cut on, $(|\alpha| \approx 1)$.

242 Simplifications to Eq. (6) for the pressure cross spectrum at the duct wall are obtained by replacing $\Psi_{m n}^{2}(\mathbf{y})$ by its average value at the duct wall ${ }^{10}$, averaged over all values of mode indices $m$ and $n$, 


$$
\Psi_{m n}^{2}(\mathbf{y})=\left\langle\Psi^{2}(\mathbf{y})\right\rangle_{m n}=2
$$

247 Taking the average incurs greatest error for modes with the largest $m$ values whose values of $\Psi_{m n}^{2}(\mathbf{y})$ are concentrated at the duct wall. These modes are comparatively scarce, however (with $m=0$ having the largest number of radial modes and hence being most common), and hence the approximation of Eq. (14) introduces negligible error compared with the exact calculation of Eq. (6). Substituting Eq. (7) for $\mid A_{m n}(\omega)^{2}$, Eq. (3a) for $k_{m n}$, and taking the high frequency limit in the sense of Eq. (10), leads to an integral expression for the pressure cross spectrum $S_{12}\left(\omega, \mathbf{y}_{a}, \Delta x\right)$ between two microphones separated axially by a distance $\Delta x$ at the duct wall $\mathbf{y}_{a}=(a, \theta)$ involving only the cutoff ratio and the frequency-dependent source strength,

$$
\frac{S_{12}\left(\hat{\omega}, \mathbf{y}_{a}\right)}{S(\hat{\omega})}=2 \mathrm{Ne}^{-i M \hat{\omega}} \int_{-1}^{1} \overline{a^{2}}(\alpha) n(\alpha) \mathrm{e}^{i \hat{\omega} \alpha} d \alpha
$$

which is a function only of the non-dimensional frequency, $\hat{\omega}$

$$
\hat{\omega}=\omega \Delta x / c \beta^{2}
$$

Note that the source term $S=S(\hat{\omega})$ has also been written as a function of $\hat{\omega}$ which is permissible since $S$ is a source term and therefore unrelated to $\Delta x$ and so there is no difficulty in non-dimensionalising the source frequency $\omega$ with respect to this arbitrary distance.

A consequence of making the separability assumption of Eq. (7) is that the cross spectrum is only a function of the non-dimensional frequency, $\hat{\omega}$. Thus, cross spectra measured at the duct wall for different separation distances $\Delta x$, plotted against $\hat{\omega}$, should collapse provided that this separability assumption is met. This property therefore provides a simple test of the validity of Eq. (7). In practice, however, the coherence measurement will be affected by non-acoustic pressure contributions from flow noise at the microphones. In 
practice, therefore, steps should be taken to minimize contamination by flow noise by, for example, recessing the microphones into the duct wall.

272

273

$$
\left[\frac{1}{A} \int_{S} S_{11}(\hat{\omega}, \mathbf{y}) d S(\mathbf{y})\right]_{f \rightarrow \infty}=S(\hat{\omega}) \mathrm{N}\left[\int_{-1}^{1} \overline{a^{2}}(\alpha) n(\alpha) d \alpha\right]\left[\frac{1}{A} \int_{A} \Psi_{m n}^{2}(\mathbf{y}) d S(\mathbf{y})\right]
$$

Noting the normalization property of the mode shape functions, $A^{-1} \int_{S} \Psi_{m n}^{2}(\mathbf{y}) d S(\mathbf{y})=1$ and the mode

$$
S(\hat{\omega})=\left[\frac{1}{\mathrm{~N}} \frac{1}{A} \int_{S} S_{11}(\hat{\omega}, \mathbf{y}) d S(\mathbf{y})\right]_{f \rightarrow \infty}
$$

The source strength $S(\hat{\omega})$ therefore has the interpretation as the high frequency noise pressure spectrum averaged over the duct cross sectional area, per mode. Joseph et al ${ }^{10}$ have shown that, in the high frequency Density (PSD) measured at the duct wall $S_{11}\left(\hat{\omega}, \mathbf{y}_{a}\right)$, i.e.,

$$
S_{11}\left(\hat{\omega}, \mathbf{y}_{a}\right)=2 \mathrm{~N} S(\hat{\omega})
$$




$$
S_{12}(\hat{\omega})=S_{11}(\hat{\omega}) \mathrm{e}^{-i M \hat{\omega}} \int_{-1}^{1} \overline{a^{2}}(\alpha)|\alpha| \mathrm{e}^{i \hat{\omega} \alpha} d \alpha
$$

In Eq. (20), and all future results, the dependence on $\mathbf{y}_{a}$ is dropped since it is now understood that all measurements are made at the duct wall.

The approximation is now made that $S_{11}(\hat{\omega}) \approx S_{22}(\hat{\omega})$ and hence $S_{11}(\hat{\omega}) \approx \sqrt{S_{11}(\hat{\omega}) S_{22}(\hat{\omega})}$, since $\Delta x$ is usually very small compared to the acoustic wavelength (typically a few centimeters). Finally the limits of integration of Eq. (20) are now extended to $\pm \infty$ based on the principle that modes for which $|\alpha|>1$ are not defined, do not exist, and may therefore be set equal to zero $\overline{a^{2}}(\alpha)=0$ for $\alpha$-values in this range. The final result is,

302

$$
\psi_{12}(\hat{\omega})=\mathrm{e}^{-i M \hat{\omega}} \int_{-\infty}^{\infty} \overline{a^{2}}(\alpha)|\alpha| \mathrm{e}^{i \hat{\omega} \alpha} d \alpha
$$

Equation (21) represents a Fourier Transform relationship between the $\alpha$ - weighted normalized mode

306 amplitude distribution function $\overline{a^{2}}(\alpha)$ and the complex coherence function $\psi_{12}(\hat{\omega})$,

307

308

$$
\psi_{12}(\hat{\omega}) \approx \frac{S_{12}(\hat{\omega})}{\sqrt{S_{11}(\hat{\omega}) S_{22}(\hat{\omega})}}\left(0 \leq\left|\psi_{12}(\hat{\omega})\right|^{2} \leq 1\right)
$$
readily deduced from the inverse Fourier Transform of the complex coherence function, weighted by $\mathrm{e}^{i M \hat{\omega}}$.

$$
\overline{a^{2}}(\alpha)=\frac{1}{2 \pi|\alpha|} \int_{-\infty}^{\infty} \psi_{12}(\hat{\omega}) \mathrm{e}^{i M \hat{\omega}} \mathrm{e}^{-i \hat{\omega} \alpha} d \hat{\omega}
$$


Equation (23) is the main result of this paper. It suggests that the normalized mode amplitude distribution may in principle be deduced using just two microphones for any incoherent multi-mode sound field whose mode amplitude distribution satisfies the separability condition of Eq. (7). The phase factor $\mathrm{e}^{i M \hat{\omega}}$ serves as a Lorentz transformation into the reference frame moving with the flow such that the amplitude distribution for right and left-traveling propagating modes is now symmetric in $\alpha$, lying in the range $-1 \geq \alpha>1$.

\section{B. Fourier Relationships}

Equations (21) and (23) are the main results of this paper. They suggest that modal cuton ratio and flowcorrected Helmholtz number, $[\alpha, \hat{\omega}]$, are conjugate variables which form Fourier transform pairs in the same way that, for example, time and frequency, $[t, f]$ are related. It is therefore not possible to specify both quantities simultaneously with arbitrary precision. By specifying certainty in the frequency spectrum and hence small values of $\Delta \hat{\omega}$, by for example imposing high resolution in the frequency spectrum, therefore imposes corresponding uncertainly $\Delta \alpha$ (or poor resolution) in the $\alpha$-spectrum, $\Delta \alpha=2 \pi / \Delta \hat{\omega}$.

The $\alpha$-spectrum is bounded by $0 \leq|\alpha| \leq 1$, with values $|\alpha|>1$ representing cutoff modes whose amplitude decay exponentially away from the source. The bandwidth $\Delta \alpha$ (appropriately defined) must therefore be less than unity and hence the coherence bandwidth (defined in a consistent way with $\Delta \alpha$ ) satisfies and hence $\Delta \hat{\omega} \Delta \alpha \approx 2 \pi$. A consequence of this band-limited behavior is that bandwidth of the coherence function is also bounded by $\Delta \hat{\omega} \approx 2 \pi$.

\section{Directivity}

In this section we explore how the two microphone method may be used to deduce the far field mean square pressure directivity $S_{f f}(\omega, \theta)$. Sinayoko et $\mathrm{al}^{12}$ have recently shown that, for the idealized case where the flow speed is uniform and everywhere the same, in the high frequency limit, there is a direct correspondence between the mean square modal distribution incident upon the open end of the duct $\overline{A_{+}^{2}}(\alpha)$ and the far field 
340 directivity. Putting Eq. (7), $E\left\{\left|A_{m n}^{+}(\omega)\right|^{2}\right\}=\frac{1}{2} S_{11}(\omega) \overline{a_{+}^{2}}\left(\alpha_{m n}\right)$, in Eq. (26) of the paper by Sinayoko et al 12 , this

341 expression may be written as

$$
\frac{S_{f f}(\omega, \theta)}{S_{11}(\omega)}=\frac{1}{4}\left(\frac{a}{R}\right)^{2} \frac{\beta^{2} \cos ^{2} \theta}{1-M^{2} \sin ^{2} \theta} \overline{a_{+}^{2}}(\alpha)
$$

where $\alpha$ is related to far field polar radiation angle $\theta$ relative to the duct axis by ${ }^{9}$,

$$
\alpha(\theta)=\frac{\cos \theta}{\sqrt{1-M^{2} \sin ^{2} \theta}} \quad(0 \leq \theta \leq \pi / 2)
$$

Substituting Eq. (23) for $\overline{a_{+}^{2}}(\alpha)$ in Eq. (24), the final result for the pressure PSD directivity $S_{f f}^{ \pm}(\omega, \theta)$ in terms

$$
\frac{S_{f f}(\omega, \theta)}{S_{11}(\omega)}=\frac{1}{8 \pi}\left(\frac{a}{R}\right)^{2} \frac{\cos \theta}{\sqrt{1-M^{2} \sin ^{2} \theta}} \int_{-\infty}^{\infty} \psi_{12}(\hat{\omega}) \mathrm{e}^{i M \hat{\omega}} \mathrm{e}^{-i \hat{\omega} \cos \theta / \sqrt{1-M^{2} \sin ^{2} \theta}} d \hat{\omega} \quad(0 \leq \theta \leq \pi / 2)
$$

Predictions of the far field directivity $S_{f f}(\omega, \theta)$ deduced from measurements of the acoustic pressure coherence function $\psi_{12}(\hat{\omega})$ have been presented in a recent paper by Melling et $\mathrm{al}^{13}$. In this work, one end of a plastic duct of length $4.34 \mathrm{~m}$ and internal dimeter $0.4 \mathrm{~m}$ was located within a reverberation chamber which was excited by four loudspeakers excited by incoherent white noise signals. The other end of the pipe was located within an anechoic chamber to allow measurements of the far field directivity function using 11 equally spaced microphones along an arc, $2.5 \mathrm{~m}$ from the duct center. These direct directivity measurements were compared at the frequencies of $3 \mathrm{kHz}$, $6 \mathrm{kHz}$ and $12 \mathrm{kHz}$ against directivity estimates deduced from the pressure coherence function at the duct wall from two microphones separated at various distances. Agreement between the directly measured directivity and the 
estimated directivity from Eq. (26) from measurements of the coherence function are generally within about $5 \mathrm{~dB}$ at any given frequency and radiation angle

\section{SOUND POWER}

The total sound power flowing along the duct provides a single measure of total noise generation. Its exact measurement in a duct system requires the amplitude of individual modes to be determined in both directions, which requires numerous microphone measurements. Alternatively, total power transmission can be determined from the integration of the far field intensity from the duct inlet or exhaust. In this section we show how upstream and downstream sound power in the mean flow can be determined solely from the complex coherenct function at the duct wall.

In a duct with uniform mean flow the sound power transmitted along the duct is the sum of the incident and reflected sound powers,

The sound power in each direction may be computed from the sum of their respective modal power components ${ }^{14}$,

$$
W^{ \pm}(\omega)=\sum_{m, n} W_{m n}^{ \pm}(\omega), \quad W_{m n}^{ \pm}(\omega)=\frac{ \pm \alpha_{m n} A\left|A_{m n}^{ \pm}\right|^{2}}{2 \rho c} \frac{\left(1-M^{2}\right)^{2}}{\left(1 \mp \alpha_{m n} M\right)^{2}} \quad\left(\alpha_{m n} \geq 0\right)
$$

Taking the high frequency limit, substituting Eq. (7) for $\left|A_{m n}\right|^{2}$ and Eq. (13) for $n(\alpha)$, noting that $S(\omega)=\frac{1}{2} S_{11}(\omega, \mathbf{y})$, and representing negative going modes by negative $\alpha$ as in Eq. (20), yields the following expression for the normalized sound power spectrum transmitted along the duct in the direction of flow, $W^{+}(\omega)$ and against the flow $W^{-}(\omega)$ of the form, 


$$
\frac{4 \pi W^{ \pm}(\omega) \rho c}{S_{11}(\omega) \beta^{4} A}=\int_{0}^{\infty} \psi_{12}(\hat{\omega}) \Omega^{ \pm}(\hat{\omega}, M) \mathrm{e}^{i M \hat{\omega}} d \hat{\omega}
$$

where

$$
\Omega^{-}(\hat{\omega}, M)=\int_{-1}^{0} \frac{\alpha \mathrm{e}^{i \hat{\omega} \alpha}}{(1-\alpha M)^{2}} d \alpha, \quad \Omega^{+}(\hat{\omega}, M)=\int_{0}^{1} \frac{\alpha e^{i \hat{\omega} \alpha}}{(1-\alpha M)^{2}} d \alpha
$$

These power factors may be evaluated in terms of standard functions,

$$
\Omega^{ \pm}(\hat{\omega})=\frac{\mathrm{e}^{i \hat{\omega} / M}}{M^{3}}\left[\mp(M+i \hat{\omega}) E i\left(\frac{-i \hat{\omega}}{M}\right) \mp M \mathrm{e}^{-i \hat{\omega} / M} \pm(M+i \hat{\omega}) E i\left(\frac{i \hat{\omega}( \pm M-1)}{M}\right)-\frac{M}{M \mp 1} \mathrm{e}^{i \hat{\omega}( \pm M-1) / M}\right]
$$

where $E i(x)$ denotes the Exponential integral function, $E i(x)=\int_{-\infty}^{x}\left(e^{t} / t\right) d t$. The final expression for the sound power spectrum $W(\omega)$ normalized on the pressure PSD at the duct wall $S_{11}(\omega)$ may therefore be written in the form of a single integral over non-dimensional frequency.

Equation (29) predicts that $W(\hat{\omega}) \rho c / A S_{11}(\hat{\omega}) \propto f(M)$, i.e., that the ratio of sound power to mean square pressure (at the duct wall) is a non-dimensional function of Mach number and is a frequency-independent constant for the class of mode amplitude distribution functions of interest in this paper. This was also the finding by Joseph et al ${ }^{10}$ in exact calculations of $W(\hat{\omega}) \rho c / S_{11}(\hat{\omega}) A$ for a variety of mode amplitude distributions, which allowed sound power to be deduced from measurements of the pressure spectrum at the duct wall providing the mode amplitude distribution was known a priori. The current method allows this constant to be determined from the coherence function which provides information about the mode amplitude distribution.

An alternative expression for the transmitted sound power may be obtained by integrating the far field intensity over a hemi-sphere with the assumption that, $(i)$ the sound power incident upon the duct exit is 
411 radiated to the far field, (ii) most of the sound power is transmitted between polar angles in the range

$4120 \leq \theta \leq \pi / 2$, i.e., with negligible radiation to the rear arc. The PSD of the radiated sound power may be

413 written in the form 6

414

415

$$
W(\omega)=\frac{4 \pi R^{2}}{\rho c} \int_{0}^{\pi / 2} S_{f f}(\omega, \theta) F(\theta) \sin \theta d \theta
$$

416

where $R$ is the distance from the duct centre at which $S_{f f}$ is evaluated and,

$$
F(\theta)=\frac{\beta^{4} \sqrt{1-M^{2} \sin ^{2} \theta}}{2\left(\sqrt{1-M^{2} \sin ^{2} \theta}-M \cos \theta\right)^{2}}
$$

Substituting Eq. (26) into (32) gives an alternative expression for the radiated sound power, which unlike Eq. (29), involves a double integration over $\omega$ and $\theta$,

$$
\frac{W \rho c}{S_{11}(\omega) a^{2}}=\frac{\beta^{4}}{16}\left(\frac{k a}{2}\right)^{2 \pi / 2} \int_{0}^{\infty} \Theta(\theta) \int_{-\infty}^{\infty} \psi_{12}(\hat{\omega}) \mathrm{e}^{i M \hat{\omega}} e^{-i \hat{\omega} \cos \theta / \sqrt{1-M^{2} \sin ^{2} \theta}} d \hat{\omega} d \theta
$$

where

$$
\Theta(\theta)=\frac{\cos \theta\left(1-M^{2} \sin ^{2} \theta\right)}{\left(1-M^{2} \cos ^{2} \theta\right)\left(\sqrt{1-M^{2} \sin ^{2} \theta}-M \cos \theta\right)^{2}}
$$

\section{NUMERICAL VALIDATION AND SPECIAL CASES}

In this section we validate the principles set out above by a number of numerical examples to illustrate the effectiveness of the technique in deducing, based only on the complex coherence measurement at the duct wall, the mode amplitude distribution and transmitted sound power for incident and reflected modes, and the 


\section{4}

55

far field pressure directivity. Further, we consider in detail some special idealized cases for more detailed analysis.

We consider the idealized case of a duct in which all the modes propagating towards the end of the duct contain equal sound power. The normalized mode amplitude distribution is obtained by setting $W_{m n}^{ \pm}(\omega)=1$ in Eq. (28) and normalizing the result according to Eq. (11b),

$$
\overline{a^{2}}(\alpha)=\frac{(1-\alpha M)^{2}}{\alpha\left(1+M^{2} / 3\right)}
$$

In a hard walled cylindrical duct the mode shape function are of the form $\left|\Psi_{m n}(r)\right|=\mid J_{m}\left(k_{r_{m n}} r\right) / \Gamma_{m n}$, where $J_{m}$ are Bessel functions of the $1^{\text {st }}$ kind or order $m, k_{r_{m n}} a$ is the $n^{\text {th }}$ stationary value of $J_{m}$ and $\Gamma_{m n}$ are constants chosen to satisfy the normalisation condition presented above. Modal pressure reflection coefficients of the form $R(\alpha)=\exp (-\delta \alpha / 2)$ are assumed in the simulations, so that $\overline{a_{-}^{2}}(\alpha)=R^{2}(\alpha) \overline{a_{+}^{2}}(\alpha)$, where $\delta$ specifies the rate at which the reflection coefficient diminishes as the modes is excited well above cut off. This reflection coefficient model is consistent with other more accurate models and is designed to ensure that modes at cutoff, $\alpha=0$, are perfectly reflected, with the reflection coefficient reducing as the modes become increasing cuton as frequency is increased ${ }^{14}$.

\section{A. Special cases; zero Mach number, arbitrary reflection}

We first consider the case of $M=0$ since it allows analytic expression to be derived and compared against exact numerical predictions. For the case of Equal energy per Mode, the mode amplitude distribution may be obtained by setting the sound power in each mode equal to unity, $W_{m n}^{+}(\omega)=W_{0}=1$ in Eq. (28),

$$
\overline{a^{2}}(\alpha)= \begin{cases}1 / \alpha N & \alpha \geq 0 \\ \exp (-\delta \alpha) /(|\alpha| N) \alpha \leq 0 & \end{cases}
$$


457 where $N$ is the factor designed to ensure that $\overline{a^{2}}(\alpha)$ is correctly normalized according to Eq. (10) and equals,

$$
N=\left(1+\delta-e^{-\delta}\right) / \delta
$$

B. Coherence function

The complex coherence function is obtained from substituting Eqs (37) into (21) to give

462

$$
\psi_{12}(\hat{\omega})=\frac{\delta}{1+\delta-e^{-\delta}}\left[\frac{\mathrm{e}^{-\delta / 2} \mathrm{e}^{-\frac{1}{2} \hat{\omega}}\left[\sin \frac{1}{2} \hat{\omega} \cosh \frac{1}{2} \delta-i \cos \frac{1}{2} \hat{\omega} \sinh \frac{1}{2} \delta\right]}{\frac{1}{2}(\hat{\omega}-i \delta)}+\frac{\mathrm{e}^{\frac{1}{2} \hat{\omega}} \sin \left(\frac{1}{2} \hat{\omega}\right)}{\frac{1}{2} \hat{\omega}}\right]
$$

464

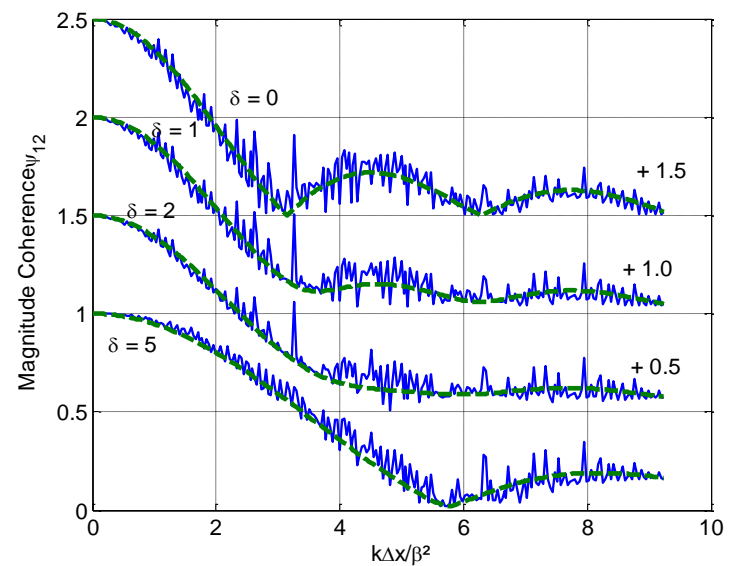

Figure 2a. Comparison of 'exact' and theoretical coherence function magnitude for $M=0$ at different levels of reflectivity, $\delta=0,1,2$ and 5 . Note that the curves have been separated for ease of viewing.

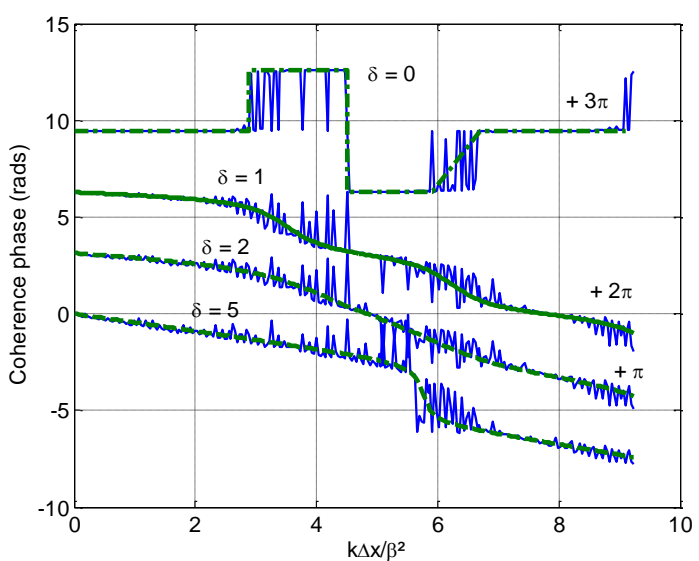

Figure 2b. Comparison of 'exact' and theoretical coherence function phase for $M=0$ at different levels of reflectivity, $\delta=0,1,2$ and 5 . Note that the curves have been separated for ease of viewing. 
Oscillations in the exact calculation arise from the behavior of the spectra at the modal cuton frequencies. Here the pressure amplitude tends to infinity as the cutoff frequency is approached. As the modal reflectivity is increased (by reducing $\delta$ ) the coherence magnitude and phase both exhibit greater variability.

Limiting cases of the coherence function may be obtained for the case of perfect modal reflectivity $\delta=0$, and when the reflectivity is zero, i.e., the duct may be assumed to be infinite. In the latter case, putting $\delta \rightarrow \infty$ into Eq. (38) yields

$$
\psi_{12}(\hat{\omega}) \rightarrow \exp \left(\frac{1}{2} i \hat{\omega}\right) \frac{\sin \left(\frac{1}{2} \hat{\omega}\right)}{\frac{1}{2} \hat{\omega}}
$$

which is in close agreeement with the exact calculation shown in figures $2 \mathrm{a}$ and $2 \mathrm{~b}$. The ducted sound field may now be regarded as a one-sided (or hemi-diffuse sound field). The phase delay between the two microphones which varies with frequency as $\frac{1}{2} \omega \Delta x / c$, i.e., precisely half the rate of a purely plane wave. Precisely this behavior is observed in figure $2 \mathrm{a}$ and $\mathrm{b}$ for the case of least reflectivity, $\delta=5$.

When all modes are perectly reflected at the end of the duct, $\delta \rightarrow 0$, and Eq. (38) tends to

$$
\psi_{12}(\hat{\omega}) \rightarrow \frac{\sin \hat{\omega}}{\hat{\omega}}
$$

In this case, where each incident mode has equal sound power and is perfectly reflected incoherently, the coherence function is identical to that of a diffuse sound field in which energy is arriving from all angles equally. Clearly, therefore, there is no phase variation between the two microphones, as shown in figure $2 \mathrm{~b}$, where $2 \pi$ phase jumps can be observed due to unwrapping issues.
C. Mode amplitude distribution 


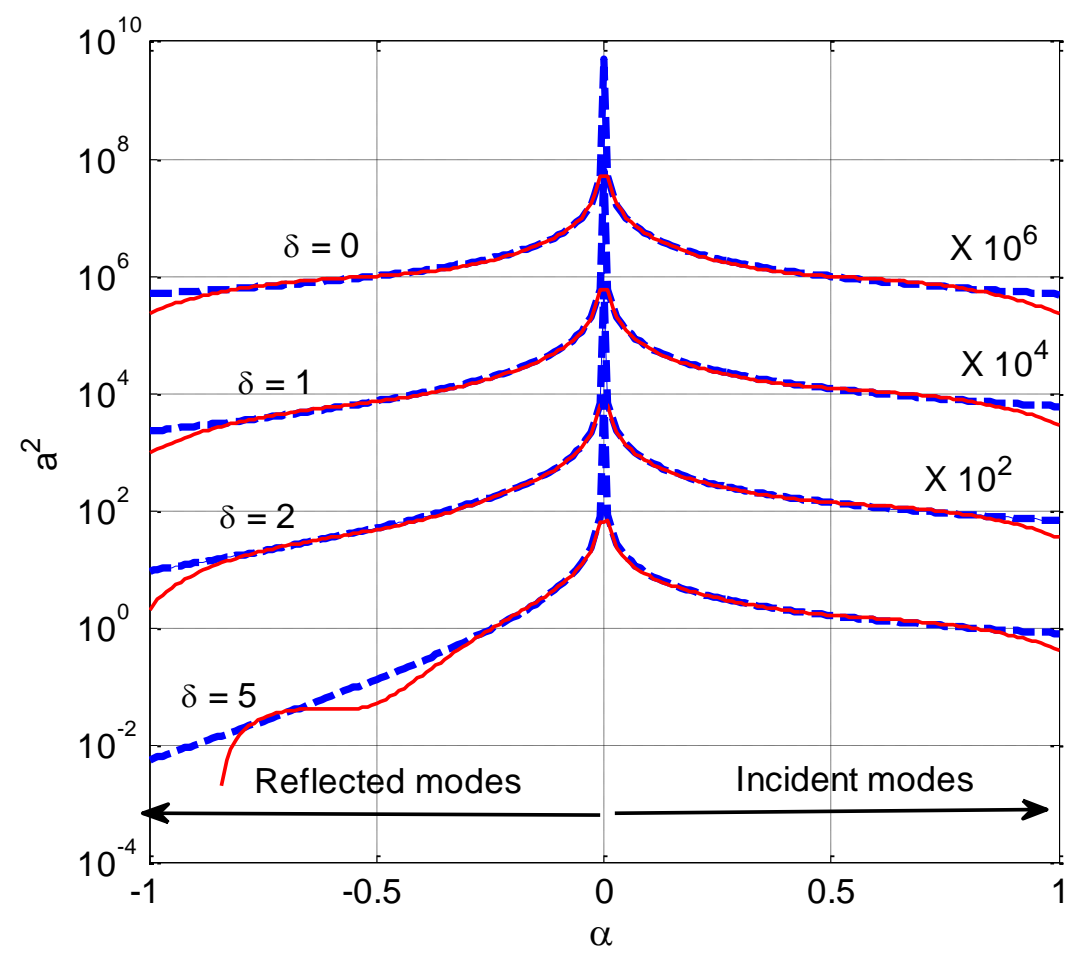

499

Figure 3. comparison of the exact (dashed curves) and inverted mode amplitude distribution (solid curves) for four reflectivity factors at $M=0$.

Agreement between the exact and inverted mode amplitude distribution is geneally excellent except near the extreme value of $\alpha=0$, where the modes are well cuton, and $|\alpha|=1$, corresponding to modes that are close to cutoff. Errors are particularly great for the very well cuton modes. This is likely to be due to the choice of 'equal energy per mode' model chosen for the simulation since the mode distribution becomes singular at $\alpha=$ 0, which clearly cannot be recovered from Eq. (23) using a numerical integration. Errors are also pronounced for the near-cutoff modes particularly for the case of least reflectivity arising from numerical errors in the evaluation of Eq. (23). 


$$
\frac{S_{f f}(\omega, \theta)}{S_{11}(\omega)}=\frac{1}{8 \pi}\left(\frac{a}{R}\right)^{2} \cos \theta \int_{-\infty}^{\infty} \psi_{12}(\hat{\omega}) \mathrm{e}^{-i \hat{\omega} \cos \theta} d \hat{\omega}
$$

515

516 Equation (39) in Eq. (41) yields the following expression for the radiated pressure

517

518

$$
\frac{S_{f f}^{ \pm}(\omega, \theta)}{S_{11}(\omega)}=\frac{1}{8 \pi}\left(\frac{a}{R}\right)^{2} \cos \theta \int_{-\infty}^{\infty} \frac{\sin \left(\frac{1}{2} \hat{\omega}\right)}{\frac{1}{2} \hat{\omega}} \mathrm{e}^{-i \hat{\omega}\left(\cos \theta+\frac{1}{2}\right)} d \hat{\omega}
$$

519

520 The integral over $\hat{\omega}$ is independent of $\theta$ and equals $2 \pi$ so that

521

522

$$
\frac{S_{f f}^{ \pm}(\omega, \theta)}{S_{11}(\omega)}=\frac{1}{4}(a / R)^{2} \cos \theta
$$

523

524 A comparison of the exact directivity (solid curve) normalised on the pressure spectrum at the duct wall

525 evaluated at the maximum frequency of interest in this calculation with that deduced from the inversion of 526 the coherence (dashed curve) is shown below in figure 4 . 


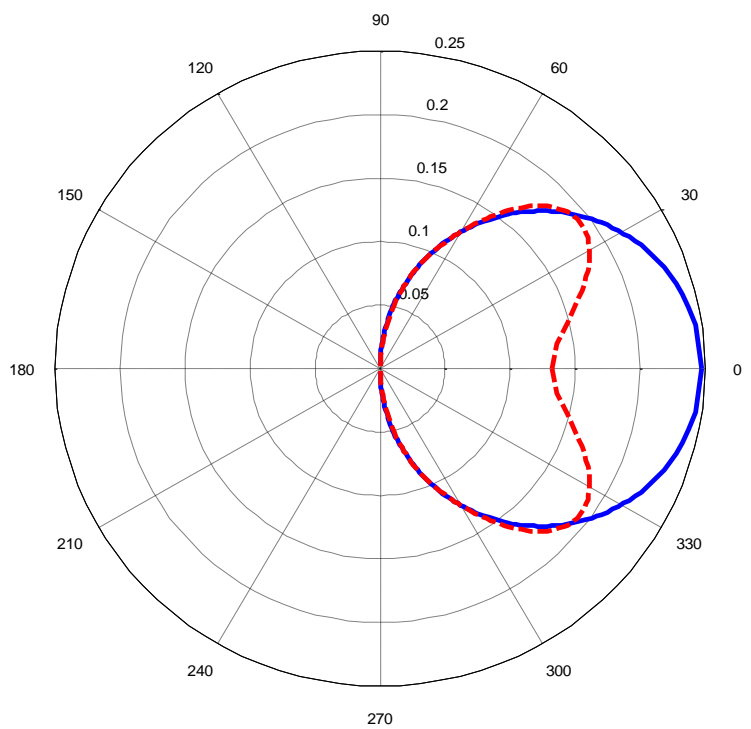

Figure 4. comparison of mean square pressure directivity at $M=0$ for $\delta=20$ obtained by inversion of the coherence function (dashed curve) and the theoretical directivity of Eq. (49) (solid curve).

Agreement is excellent at radiation angles beyond about $45^{\circ}$ to the duct axis but then deviates at smaller angles close to the duct axis, incurring a maximum error on-axis of about $40 \%$ in the pressure amplitude

534 (4.4dB in sound pressure level). Radiation within this range of angles is due to well cut-on modes with $\alpha-$ values close to 1, as also observed in Fig 3 in which greatest error in the mode amplitude distribution is for $\alpha$

$536 \sim 1$, whose main radiation lobes are close to the duct axis. The reason for the error in these modes is due to 537 the Gibbs phenomenon in Fourier analysis, in which a 'ringing' type behaviour is observed in the vicinity of piecewise discontinuities, such as in the present case. Here, the mode amplitude distribution is discontinuous at $\alpha=1$, since the measurement location is assumed to be well away from the source so that no cutoff modes

540 are present in the sound field, i.e., $\overline{a^{2}}(\alpha)=0$ for $\alpha>1$. 
546 Figure $5 \mathrm{a}$ and $5 \mathrm{~b}$ show the variation of upstream and downstream sound power versus $\hat{\omega}$ for the cases of 547 zero reflection $(\delta=20)$ and perfect reflection, respectively. The dashed curve represents the variation computed from the exact expression of Eq. (28) while the solid curve is that obtained by inversion of the coherence function using Eq. (23).

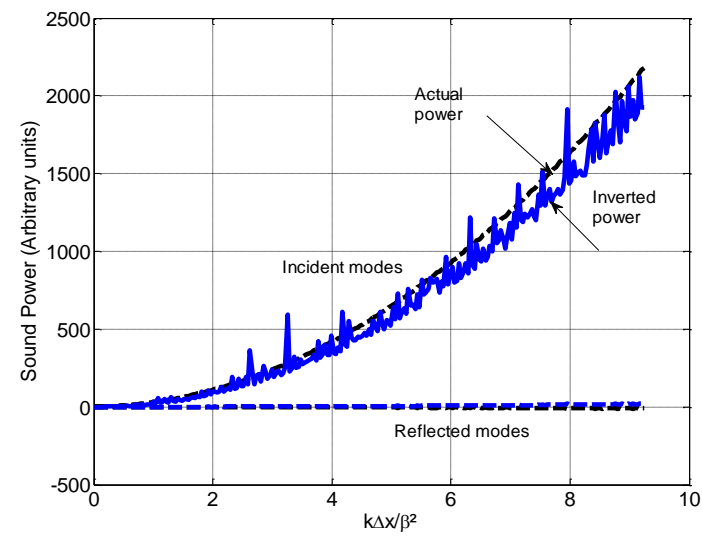

Figure 5a. Comparison of actual (solid curve) and inverted (dashed curve) upstream and downstream sound power for $M=0$ and $\delta=20$.

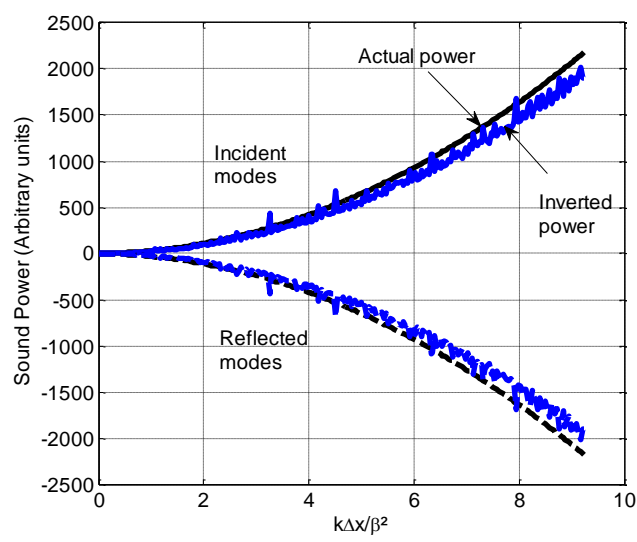

Figure 5b. Comparison of actual (solid curve) and inverted (dashed curve) upstream and downstream sound power for $M=0$ and $\delta=0$.

Whilst this frequency variation is unrealistic since equal sound power per mode is assumed and each mode is assumed to contain unit sound power the agreement observed above is useful for validating the measurement principle. The power variation of the incident modes (before reflection) is therefore identical to that of the variation of the number of modes. Thus, $W^{+}(\hat{\omega})=\left(\frac{1}{2} k a\right)^{2} W_{0}$, where $W_{0}=1$.

$$
\frac{4 \pi W^{ \pm}(\omega) \rho c}{S_{11}(\omega) A}=\int_{0}^{\infty} \frac{e^{i \frac{1}{2} \hat{\omega}} \sin \left(\frac{1}{2} \hat{\omega}\right)}{\frac{1}{2} \hat{\omega}} \frac{\left[e^{i \hat{\omega}}(1 \mp i \hat{\omega}) \mp 1\right]}{\hat{\omega}^{2}} d \hat{\omega}
$$


564 The integral on the right of Eq. (44) equals $\pi$ and hence, the sound power transmitted along the duct and 565 hence radiated to the far field may be computed from,

566

567

568

569

570

571

572

573

574

575

576

577

$$
S_{f f}(\theta)=\frac{1}{2}\left(\frac{2 \rho c W_{0}}{A}\right)\left(\frac{a}{R}\right)^{2}\left(\frac{k a}{2}\right)^{2} \frac{\left(\sqrt{\left(1-M^{2} \sin ^{2} \theta\right)}-M \cos \theta\right)^{2} \cos \theta}{\left(1+\frac{1}{3} M^{2}\right)\left(1-M^{2} \sin ^{2} \theta\right)^{5 / 2}}
$$

$$
W(\omega)=\frac{A S_{11}(\hat{\omega})}{4 \rho c}
$$

This relationship between transmitted sound power and mean square pressure at the duct wall for the case of an 'equal energy per mode' sound field has previously derived using an alternative method by Joseph et $\mathrm{al}^{4}$.

\section{F. Special cases: Perfect reflection ( $\delta \rightarrow 0)$, zero Mach number $(M \rightarrow 0)$}

For completeness we consider the unrealistic case in which all propagating modes are reflected back into the duct without attenuation.

For the case of the perfectly reflecting duct, substituting Eq. (40) for the coherence function into the expression for sound power, Eq. (29), gives $W^{ \pm}(\omega) \rightarrow 0$ and $S_{f f}(\omega, \theta) \rightarrow 0$. Thus, sound is perfectly reflected from the open end and hence there is no net transmitted sound power and hence no far field radiation.

At moderately high frequency and above $(k a>10)$ modal reflections are weak except for all modes except those excited close to their cut off frequency, where $|\alpha| \approx 1$. In this case we may set $\delta \rightarrow \infty$ and the normalized mode amplitude distribution is given by Eq. (36). From Sinayoko et al ${ }^{6}$ it may be shown that 
Putting (46) into (20) and taking the high frequency limit

$$
S_{11}=4\left(\frac{1}{2} k a / \beta\right)^{2} 2 \rho c W_{0} / A
$$

So that the ratio of mean square far field pressure to that at the duct wall is

$$
\frac{S_{f f}(\theta)}{S_{11}}=\frac{\beta^{2}}{4}\left(\frac{a}{R}\right)^{2} \frac{\left(\sqrt{\left(1-M^{2} \sin ^{2} \theta\right)}-M \cos \theta\right)^{2} \cos \theta}{\left(1+\frac{1}{3} M^{2}\right)\left(1-M^{2} \sin ^{2} \theta\right)^{5 / 2}}
$$
coherence function calculated for $M=0.5$ and $\delta=20$ (not shown here) in figure 6 .

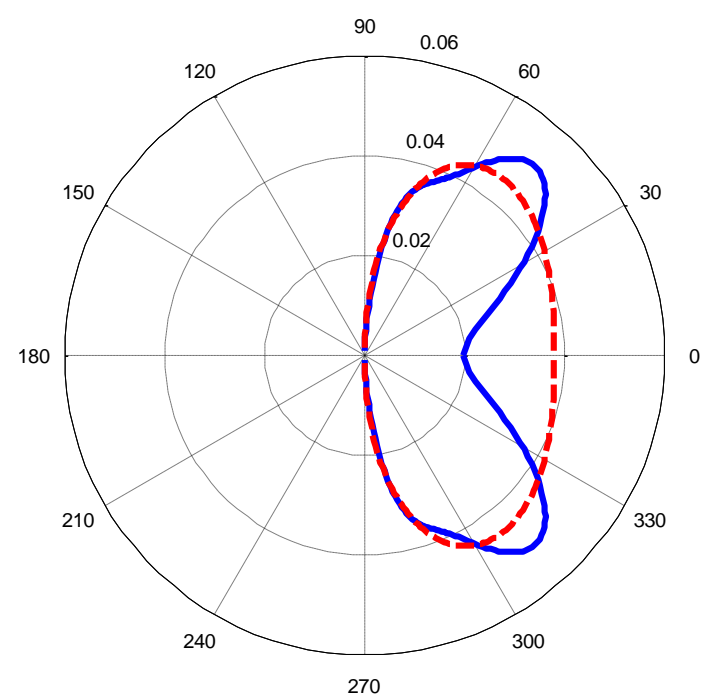

599 Figure 6. comparison of mean square pressure directivity at $M=0.5$ for $\delta=20$ obtained by inversion of the 600 coherence function (solid curve) and the theoretical directivity of Eq. (49) (dashed curve) As for the case of zero Mach number good agreement is observed at sideline angles with greatest incurred for the angles close to the duct axis. The most pronounced effect of non-zero Mach number is that the directivity 
is now 'flattened' with now most energy radiated to the sideline directions. This behavior is captured by the inverted directivity function.

606

This relationship between transmitted sound power and the acoustic pressure PSD evaluated at the duct wall for an equal energy per mode sound has been previously derived by Joseph et al ${ }^{10}$ and provides a useful estimate for the sound power in ducted broadband sound fields whose mode distribution is unknown.

Finally, we present the coherence function and inverted mode amplitude distribution function for the case of arbitrary Mach number and reflectivity. Figures $7 \mathrm{a}$ and $\mathrm{b}$ below show the coherence function for the more general case of $M=0.5$ and $\delta=2$, where no analytic solution is available. The corresponding mode amplitude distribution obtained from inversion of this coherence function using Eq. (23) compared with the exact distribution is plotted in figure 7c, where again good agreement is observed.

617

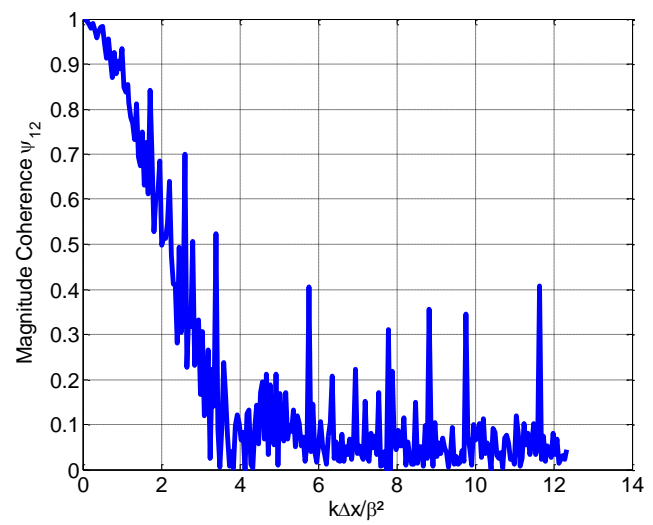

Figure 7a. Coherence function magnitude for $M$ $=0.5$ and $\delta=2$.

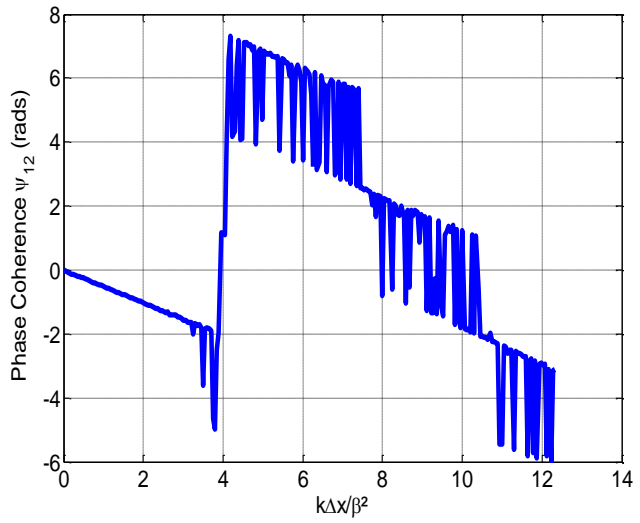

Figure 7b. Predicted coherence (dashed curve) function phase for $M=0.5$ and $\delta=2$. 


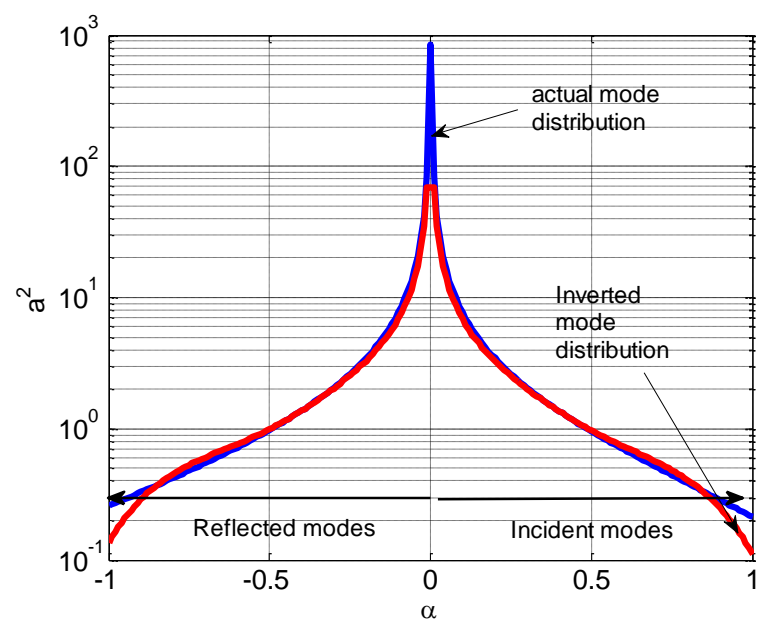

Figure 7c. comparison of the exact (solid curves) and inverted mode amplitude distribution (dashed curves) for four reflectivity factors.

\section{CONCLUSION AND DISCUSSION}

This paper has described a new method for determining the mode amplitude distribution versus modal cuton ratio in multi-mode broadband sound field in ducts in the presence of uniform mean flow and reflections. The novelty of the technique is that it requires only measurements of the complex coherence function made at the duct wall. The measurement method makes the four important assumptions about the sound field:

1. The mode amplitude distribution is separable in the form, $E\left\{\left|A_{m n}^{ \pm}(\omega)\right|^{2}\right\}=S(\omega) \overline{a_{ \pm}^{2}}\left(\alpha_{m n}\right)$

2. All propagating modes are mutually incoherent.

3. The number of modes that are able to propagate is sufficiently high that the mode distribution versus $\alpha$ may be regarded as a continuous function and the modal sum replaced by an integration.

4. Mean flow is an axial 'plug 'flow'.

Assumption 1 is satisfied for any spatial distribution of sources ${ }^{10,14}$, while assumption 2 is valid for stochastic sources with length-scales much shorter than the acoustic wavelength. Examples of the latter are fan broadband noise generated through the interaction between outlet guide vanes and rotor wake turbulence, and the noise generated by unsteady combustion. Our experience has demonstrated that Assumption 3 is sufficiently valid at non-dimensional frequencies of $k a>10$, in which there are at least 30 propagating modes. In practical cases, this condition corresponds to a frequency of about $1.1 \mathrm{kHz}$ for a duct 
644 diameter of $1 \mathrm{~m}$ and above $22 \mathrm{kHz}$ (beyond the audible range) for a duct diameter of $0.05 \mathrm{~m}$. The method is 645 therefore appropriate to relatively large duct diameters, such as turbofan engines and large exhaust stacks. 646 Assumption 4 will never be strictly satisfied in flow ducts due to the presence of boundary layers at the duct 647 wall. In these cases, the relationship between the modal axial wavenumber and $a$ in Eqs. (3) will be more 648 complicated and must be determined from the modal eigenvalues determined from numerical methods. 649 However, it is unlikely that axial flow speed variations of less than about $20 \%$ will significantly alter the 650 variation of mode amplitude with $a$, although more work is needed to confirm this assumption and extend the 651 measurement method to realistic flow profiles.

652 One practical difficulty with making the accurate coherence measurements of the acoustic field is the presence of extraneous noise due to flow passing over the microphones. A well-known method to reduce the 655 effects of flow noise on duct wall measurements is to recess the microphones ${ }^{15}$. Subsonically convecting flow 656 disturbances will radiate to the recessed microphones as evanescent waves while acoustic noise will reach 657 the microphones un-attenuated.

658 
660

661

662

663

664

665

666

667

668

669

670

671

672

673

674

675

676

677

678

679

680

681

682

683

684

685

686

687

688

689

690

691

692

693

694

695

696

697

698

699

700

701

702

703

${ }^{1}$ P. Joseph, "A Coherence Approach to Characterizing Broadband Sound Fields in Ducts," InterNoise 2014, 16 ${ }^{\text {th }}$ $19^{\text {th }}$ November, Melbourne, Australia.

${ }^{2}$ C. Moore, C., "Measurement of Radial and Circumferential Modes in Annular and Circular Ducts," Journal of Sound and Vibration, Vol. 62, No. 2, 1979, pp. 235-256.

${ }^{3}$ R. H. Thomas, F. Farassat, L. R. Clark, C. H. Gerhold, J. J. Kelly, and L. E. Becker. "A mode detection method using the azimuthal directivity of a turbofan model," AIAA paper 99-1954, 1999.

${ }^{4} \mathrm{~F}$. Farassat, D. Nark, and H. Russel, "The detection of radiated modes from ducted fan engine," in the Seventh AIAA/CEAS Aeroacoustics Conference, Maastricht, The Netherlands, 2001, Paper No. AIAA 2001-2138.

${ }^{5}$ S. Lewy, "Inverse method predicting spinning modes radiated by a ducted fan from free-field measurements," J. Acoust. Soc. Am. 117, 744-750_2005_.

${ }^{6}$ U. Tapken, T. Raitor, L. “Enghardt. Tonal Noise Radiation from an UHBR Fan Optimized In-Duct Radial Mode Analysis." AIAA 2009-3288. 15th AIAA/CEAS Aeroacoustics, 11 - 13 May 2009, Miami, Florida.

${ }^{7} \mathrm{M}$. Abom, "Modal decomposition in ducts based on transfer function measurements between microphone pairs" Journal of Sound and Vibration 135(1):95-114 (1989).

${ }^{8}$ C. R. Lowis, P. F. Joseph, A. J. Kempton, "Estimation of the far-field directivity of broadband aeroengine fan noise using an in-duct axial microphone array." Journal of Sound and Vibration, 329, Issue 19, p. 3940-3957 (2010)

${ }^{9}$ E. J. Rice, "Multimodal far-field acoustic radiation pattern using mode cutoff ratio", AIAA Journal. 16, 906-911 (1978)

${ }^{10} \mathrm{P}$. Joseph,, C.L. Morfey, C.R. Lowis, "Multi-mode sound transmission in ducts with flow", Journal of Sound and Vibration 264 523-544 (2003).

${ }^{11} \mathrm{P}$. Joseph and C. L. Morfey, "Multi-mode radiation from an unflanged, semi-infinite circular duct", Journal of the acoustical society of America 105(5), $2590-2600$ (1999).

${ }^{12}$ S. Sinayoko, P. Joseph and A. McAlpine, "Multi-mode radiation from ducts with flow", Journal of the acoustical society of America. 127(4) (2010).

${ }^{13}$ J. Melling, J. Chen, P. Joseph, "Experimental investigation of a new two-microphone method for the determination of broadband noise radiation from ducts"Applied Acoustics 117 (2017) 66-75.

${ }^{14} \mathrm{C}$.L. Morfey, "Sound transmission and generation in ducts with flow", Journal of Sound and Vibration 14, $37-55$ (1971).

${ }^{15}$ V. Fleury, L. Coste, R. Davy, A. Mignosi, C. Cariou, and J.-M. Prosper, "Optimization of Microphone Array WallMountings in Closed-Section Wind Tunnels". AIAA 2010-3738, 16th AIAA/CEAS Aeroacoustics Conference (2010). 